Pacific Journal of Mathematics

ON MINIMAL COMPLEXES 


\section{ON MINIMAL COMPLEXES}

\section{JOSEPH ZAKS}

An $n$-complex $K$ is called p.w.l. minimal in $E^{d}$ if each proper subcomplex of $K$ is p.w.l. is embeddable in $E^{d}$. The main purpose of this paper is to prove that for each $n \geqq 2$, and each $d, n+1 \leqq d \leqq 2 n$, there are countably many nonhomeomorphic $n$-complexes, each one of which is p.w.1. minimal in $E^{d}$ and is not p.w.l. embeddable there. From general position arguments it follows that if an $n$-complex $K$ is p.w.l. minimal in $E^{2 n}$, then for each $x \in|K|,|K|-\{x\}$ is embeddable topologically in $E^{2 n}$; if an $n$-complex $K$ is p.w.l. minimal in $E^{n+d}$ and is not embeddable there, then the dimension of each maximal simplex of $K$ is at least $d$.

Here $E^{d}$ denotes the Euclidean $d$-space, an $n$-complex is a finite $n$-dimensional simplicial complex. $|K|$ denotes the underlying point set of the complex $K$ in some $E^{d}$, and in case where there is no confusion, $|K|$ will be replaced by $K . \quad C_{m}^{n}$ denotes the complete $n$ complex with $m$ vertices.

A subset $X$ of $E^{d}$ is called cellular if there exists a sequence $\left\{Q_{i}\right\}_{i=1}^{\infty}$ of closed $d$-cells, such that $Q_{i+1} \subset \operatorname{Int} Q_{i}$, for each $i$, and $X=$ $\bigcap_{i=1}^{\infty} Q_{i}$; where Int means interior.

A cellular decomposition $G$ of $E^{n}$ is an upper semicontinuous (u.s.c.) decomposition of $E^{n}$, such that each element of $G$ is cellular; an u.s.c. decomposition is finite if it has only finitely many nondegenerate elements, see [1].

2. There are precisely two 1-complexes which are p.w.l. minimal in $E^{2}$ and are not topologically embeddable there: these are the two Kuratowski's nonplanar graphs, [6].

B. Grünbaum proved in [3] that all the $n$-complexes of certain form are not embeddable in $E^{2 n}$, and that one of them, for each $n$, is geometrically minimal in $E^{2 n}$, where the geometrically minimal in $E^{d}$ means that each proper subcomplex can be rectilinearly (= affine on each simplex) embedded in $E^{d}$. All of these $n$-complexes were proved by J. Zaks, in [10], to be p.w.l. minimal in $E^{2 n}$, and in certain cases, for each $n$, to be geometrically minimal there. B. Grünbaum proved in [4] that, indeed, each one of these $n$-complexes is geometrically minimal in $E^{2 n}$.

However, the number of these $n$-complexes is finite, for each $n$. Related to these results, we have the following. 
THEOREM 1. For each $n \geqq 2$, there are countably many nonhomeomorphic n-complexes, each one of which is p.w.l. minimal in $E^{2 n}$ and is not p.w.l. embeddable there.

Moreover, we extend this to a

COROLLARY 1. For each $n \geqq 2$ and each $d, n+1 \leqq d \leqq 2 n$, there are countably many nonhomeomorphic n-complexes, each one of which is p.w.l. minimal in $E^{d}$ and is not p.w.l. embeddable there.

3. Proof of Theorem 1. For the proof of this theorem, we need certain lemmas, which seem to be obvious; our proofs make use of some heavy techniques from combinatorial topology, see [8], [9] and [11].

Lemma 1. A polyhedral disk $D$ in $E^{n}$ is cellular.

Proof. Let $K$ be a triangulation of $D$. There exists a triangulation $T$ of $E^{n}$ and a subdivision $K^{1}$ of $K$ such that $K^{1}$ is a subcomplex of $T$. The disk $D$ with the triangulation $K^{1}$ can be shelled, by [8], hence $K^{1}$ collapses to a triangle, and therefore $K^{1}$ is collapsible, see [9], [11]. Using a theorem of J.H.C. Whitehead, [9], it follows that $\operatorname{st}\left(\beta^{2} K^{1}, \beta^{2} T\right)$ - the star of $K^{1}$ in $T$, taken in the second barycentric subdivision of $T$-is an $n$-cell. Let $Q_{i}=\operatorname{st}\left(\beta^{2 i} K^{1}, \beta^{2 i} T\right)$, then all the $Q_{i}-s$ are $n$-cells, $Q_{i+1} \subset \operatorname{Int} Q_{i}$ and $D=\bigcap_{i=1}^{\infty} Q_{i}$ : Therefore $D$ is cellular.

Lemma 2. If $G$ is a finite cellular decomposition of $E^{n}$, then the decomposition space $E^{n} / G$ of $G$ is homeomorphic to $E^{n}$.

This lemma is a particular and simple case of L. V. Keldysh's Theorem 1 of [5], because of the finiteness of $G$. We would like to mention the difference between the usual definition of cellularity, and that of [5]. Theorem 1 of [5] was proved later as part of Theorem 1.4 of [7].

It follows from Lemma 2 that if $\alpha$ is a polyhedral simple (closed-) arc in the interior of an $n$-simplex $\delta^{n}$ in $E^{d}$, then the space obtained from $\delta^{n}$ by shrinking $\alpha$ to a point $\left("{ }^{\prime \prime} \delta^{n}\right.$ modulo $\left.\alpha^{\prime \prime}\right)$ is homeomorphic to $\delta^{n}$. This will be stated as

Lemma 3. Let $\delta^{n}$ be an n-simplex in $E^{d}$, and let $G$ be a finite u.s.c. decomposition of $\delta^{n}$ having only polyhedral simple ares in Int $\delta^{n}$ for its nondegenerate elements, then $\delta^{n} / G$ is homeomorphic to $\delta^{n}$. 
Lemma 4. For each $n, C_{2 n+3}^{n}$ is not embeddable topologically in $E^{2 n}$; however, there exists a maps $f: C_{2 n+3}^{n} \rightarrow E^{2 n}$, which is affine on each simplex, and has only one inverse set, which contains only two points. (An inverse set of a map $f: X \rightarrow Y$ is $f^{-1}(f(x))$, provided $f^{-1}(f(x)) \neq\{x\}$.)

The nonembeddability of $C_{2 n+3}^{n}$ in $E^{2 n}$ is a well known result, due to $A$. Flores [2], and the map $f$ is described in [3], [4] (see also [10]).

Lemma 5. For each $n$ and each point $x \in\left|C_{2 n+3}^{n}\right|,\left|C_{2 n+3}^{n}\right|-\{x\}$ is embeddable in $E^{2 n}$.

This lemma will later be extended, see Theorem 2.

Proof. In the case where $x$ is an interior point of some $n$-simplex, we can use the map $f$ as given in Lemma 4. Otherwise, let $V_{x}$ be a small neighborhood of $x$ in $C_{2 n+3}^{n}$. By pushing each point of $V_{x}-\{x\}$ away from $x$, it follows that $\left|C_{2 n+3}^{n}\right|-\{x\}$ is homeomorphic to a subset of $\left|C_{2 n+3}^{n}\right|-\{y\}$, where $y$ is an interior point of some $n$-simplex, hence, by the first part of this proof, $\left|C_{2 n+3}^{n}\right|-\{y\}$ is embeddable in $E^{2 n}$, and therefore $\left|C_{2 n+3}^{n}\right|-\{x\}$ is embeddable there, too. This completes the proof of Lemma 5 .

Proof of Theorem 1. For each $n \geqq 2$, let us first define inductively a sequence $\left\{K^{n}(m)\right\}_{m=1}^{\infty}$ of $n$-complexes as follows: let $\delta^{n}$ be a fixed $n$-simplex of $C_{2 n+3}^{n}$. $K^{n}(1)$ is obtained from $C_{2 n+3}^{n}$ as follows:

Step 1. Subdivide $C_{2 n+3}^{n}$ in such a way that $\delta^{n}$ will contain as a subcomplex a simple are $A_{1}^{n} A_{2}^{n} A_{3}^{n} A_{4}^{n}$, consisting of three edges, all of them in Int $\delta^{n}$, and both of $A_{1}^{n}$ and $A_{4}^{n}$ are in the star of no vertex in the new complex.

Step 2. Identify $A_{1}^{n}=A_{4}^{n}$,

Step 3. Add a new triangle $B$, having the new circuit $A_{1}^{n} A_{2}^{n} A_{3}^{n}$ as its boundary.

$K^{n}(m)$ is obtain from $K^{n}(m-1)$ by a similar way, where we pick the new arc of Step 1 to be disjoint from all the previously added triangles $B$ of Step 3, and keep the triangles $B$ of Step 3 untouched.

Since $n \geqq 2$, and we add only 2 -simplexes, $K^{n}(m)$ is an $n$-complex.

Main claim. For each $m, K^{n}(m)$ is not p.w.l. embeddable in $E^{2 n}$.

Proof. Suppose this is false, then for some $n$ and some $m$ we have a p.w.l. embedding $f$ 


$$
f: K^{n}(m) \rightarrow E^{2 n} \text {. }
$$

Let $B_{1}, \cdots, B_{m}$ be the added triangles of $K^{n}(m)$, as described in Step 3 , and let $G$ be the decomposition of $E^{2 n}$, having $f\left(B_{i}\right), 1 \leqq i \leqq$ $m$, as the only non-degenerate elements.

By Lemma 1 , each $f\left(B_{i}\right)$ is cellular in $E^{2 n}$, since $f$ is a p.w.l. embedding; therefore $G$ is a cellular decomposition of $E^{2 n}$, and it is finite, hence by Lemma 2 there exists a homeomorphism $h: E^{2 n} / G \rightarrow E^{2 n}$. Let $p: E^{2 n} \rightarrow E^{2 n} / G$ be the natural projection, related to the decomposition $G$.

Let $g: C_{2 n+3}^{n} \rightarrow K^{n}(m)$ be the map which identifies the $m$ pairs of points, as described in Step 2, and is the identity elsewhere.

In the following diagram

$$
C_{2 n+3}^{n} \stackrel{g}{\longrightarrow} K^{n}(m) \stackrel{f}{\longrightarrow} E^{2 n} \stackrel{p}{\longrightarrow} E^{2 n} / G \stackrel{h}{\longrightarrow} E^{2 n},
$$

the map $p f g$ shrinks the $m$ polygonal simple arcs, as described in Step 1, each one to a point, hence $p f g\left(\delta^{n}\right)$ is an $n$-cell, by Lemma 3, therefore $p f g\left(C_{2 n+3}^{n}\right)$ is homeorphic to $C_{2 n+3}^{n}$, and as a result $h p f g\left(C_{2 n+3}^{n}\right)$ is a subset of $E^{2 n}$ which is homeomorphic to $C_{2 n+3}^{n}$. This contradicts Lemma 4, and hence completes the proof of the main claim.

Next, for each $n \geqq 2$, let $\left\{\widetilde{K}^{n}(m)\right\}_{m=1}^{\infty}$ be the sequence, obtained from $\left\{K^{n}(m)\right\}_{m=1}^{\infty}$ as follows: if $K^{n}(m)$ is p.w.l. minimal in $E^{2 n}$, we let $\widetilde{K}^{n}(m)=K^{n}(m)$; otherwise we define $\widetilde{K}^{n}(m)$ to be a subcomplex of $K^{n}(m)$ which is not p.w.l. embeddable in $E^{2 n}$, and is p.w.l. minimal there. Using Lemmas 4 and 5, and the fact that our construction of $K^{n}(m)$ from $C_{2 n+3}^{n}$ can be performed in a small neighborhood of any point of $C_{2 n+3}^{n}$, it follows that the only simplexes of $K^{n}(m)$ which are not in $\widetilde{K}^{n}(m)$ are triangles, among the ones added in Step 3. In particular, no point, which is the identification of two points of $\delta^{n}$, by Step 2, can be deleted. These $m$ points of $\widetilde{K}^{n}(m)$ have neighborhoods which are topologically different from neighborhoods of other points of $\widetilde{K}^{n}(m)$; therefore if $m \neq m^{\prime}, \widetilde{K}^{n}(m)$ and $\widetilde{K}^{n}\left(m^{\prime}\right)$ are not homeomorphic, and the proof of Theorem 1 is completed.

From Corollary 2 it will follow that for $n \geqq 3$, no one of the $m$ added triangles of $K^{n}(m)$, by Step 3 , appears in $\widetilde{K}^{n}(m)$, and $\widetilde{K}^{n}(m)$ is just the result of identifying $m$ pairs of points in Int $\delta$, each pair to a point. Probably, this is the case for $n=2$, too.

In order to obtain other $n$-complexes, each one of which is p.w.l. minimal in $E^{2 n}$ and is not p.w.l. embeddable there, for $\mathrm{n} \geqq 2$, we can use more than just one $n$-simplex of $C_{2 n+3}^{n}$, or we can take, to begin with, any other $n$-complex from the list in [3], since they all share the needed properties that $C_{2 n+3}^{n}$ does, by [3], [4], [10] and Theorem 2, here. Moreover, we can identify more than two point in our Step 
2 , but then we have to make some obvious alterations.

Proof of Corollary 1. Let us first observe that if a complex $K$ is not p.w.l. embeddable in $E^{d}$, then $K V C_{1}^{0}$ is not p.w.l. embeddable in $E^{d+1}$ and $K V C_{n}^{n-1}$ is not p.w.l. embeddable in $E^{d+n}$, where $K V L$ is the join complex of $K$ and $L$, see [11]. Moreover, if $K$ is p.w.l. minimal in $E^{d}$, then $K V C_{1}^{0}$ is p.w.l. minimal in $E^{d+1}$, by [10], and therefore $K V C_{n}^{n-1}$ is p.w.l. minimal in $E^{d+n}$.

Let $n$ and $d$ be given, where $n \geqq 2$ and $n+2 \leqq d \leqq 2$. A sequence $\left\{L^{n ; d}(m)\right\}_{m=1}^{\infty}$ of nonhomeomorphic $n$-complexes, each one of which being p.w.l. minimal in $E^{d}$ and not p.w.l. embeddable there, can be obtained as follows: $L^{n ; d}(m)=\widetilde{K}^{d-n}(m) V C_{2 n-d}^{2 n-d-1}$, where $\widetilde{K}^{d-n}(m)$ is given by Theorem 1 , which is applicable since $d-n \geqq 2$.

$L^{n ; d}(\mathrm{~m})$ is an $n$-complex, because $(d-n)+(2 n-d-1)+1=n$; it is not p.w.l. embeddable in $E^{d}$, because $\widetilde{K}^{d-n}(m)$ is not p.w.l. embeddable in $E^{2(d-n)}$, and $2(d-n)+(2 n-d)=d$.

For the case where $n \geqq 2$ and $d=n+1$, it is obviously enough to deal with $n=2$ and $d=3: L^{2: 3}(m)$ is the following complex: We take a triangulated orientable closed 2-manifold of genus $m$, which contains the shap " $X$ " as a subcomplex, having $O A, O B, O C$ and $O D$ as edges, where $A, B, C, D$ are in a clockwise order. We add two new vertices $P$ and $Q$, the four triangles $P O A, P O C, Q O B, Q O D$, together with their faces, and we add the edge $P Q$. It is very easy to verify that $L^{2 ; 3}(m)$ is not p.w.l. embeddable in $E^{3}$, and that it is p.w.l. minimal there. (Moreover, if $x$ is an interior point of one of the added triangles, then $L^{2,3}(m)-\{x\}$ is still not embeddable in $E^{3}$. Compare this comment with Theorem 2.)

\section{The following is an extension to Lemma 5:}

THEOREM 2. If an n-complex $K$ is p.w.l. minimal in $E^{2 n}$, then for each point $x \in|K|,|K|-\{x\}$ is embeddable in $E^{2 n}$.

Proof. As it was shown in the proof of Lemma 5, we can assume, without loss of generality, that $x \in \operatorname{Int} \delta$, where $\delta$ is a maximal simplex of $K$. Since $K$ is p.w.l. minimal in $E^{2 n}$, let $f:|K-\delta| \rightarrow E^{2 n}$ be a p.w.l. and general position embedding. Let $A \in E^{2 n}$ be in general position with respect to $f(|K-\delta|)$.

Let $F:|K| \rightarrow E^{2 n}$ be defined as follows

$$
F(z)= \begin{cases}f(z) & \text { if } z \in|K-\delta| \\ \lambda A+(1-\lambda) f\left(z^{\prime}\right) & \text { if } z \in \operatorname{Int} \delta \text { and } z=\lambda b_{\dot{o}}+(1-\lambda) z^{\prime}, \\ & \text { where } b_{\delta} \text { is the barycenter of } \delta, \\ & \text { and } z^{\prime} \in|B d \delta|, \quad 0<\lambda \leqq 1\end{cases}
$$


$F$ is a well defined immersion (= locally embedding) of $|K|$, and its singularities are those of $F(\operatorname{Int} \delta)$, together with the possible intersections of $F(|\delta|)$ with $F(|K-\delta|)$. Let $s$ be the dimension of $\delta$, then from general position arguments it follows that the dimensions of these singularities are either $\leqq 2 s-2 n$ or $\leqq s+t-2 n$, for some $1 \leqq t \leqq n$, and since $\mathrm{s} \leqq n$, they are $\leqq 0$. Hence the singularities of $F$ consists of finitely many points, each point $z$ of which has at least one point of $F^{-1}(z)$ in Int $\delta$.

Therefore, there exists a $t, 0<t<1$, such that $F$ is an embedding when restricted to

$$
|K|-\left\{\lambda b_{\delta}+(1-\lambda) x \mid t<\lambda \leqq 1 \text { and } x \in|B d \delta|\right\},
$$

which is homeomorphic to $|K|-\{x\}$, and the proof is completed.

Corollary 2. If an n-complex $K$ is p.w.l. minimal in $E^{n+d}$ and is not embeddable there, then the dimension of each maximal simplex of $K$ is at least $d$.

In particular, if $d=n$, then these dimensions are equal to $n$.

Proof. Let $\delta$ be a maximal $s$-simplex of $K$, which among all the maximal simplexes of $K$ is of minimal dimension.

Let $F:|K| \rightarrow E^{n+d}$ be the extension of a p.w.l. and general position embedding of $K-\delta$ in $E^{n+d}$, similar to the one described in the proof Theorem 2. The dimensions of the singularities of $F$ are $\leqq s+t-(n+d)$, with $s \leqq t \leqq n$; however it is never $\leqq-1$ because $K$ is not embeddable in $E^{n+d}$. Therefore

$$
\min _{s \leqq t \leqq n}[s+t-(n+d)]=2 s-n-d \geqq 0
$$

and since $s \leqq n$ it follows that $s \geqq d$, and the proof is completed.

The author of this paper wishes to express his grateful acknowledge to Professor Branko Grünbaum for many helpful discussions and, in particular, for his excellent encouragement.

\section{REFERENCES}

1. S. Armentrout, Monotone decompositions of $E^{3}$, Topology Seminar, Wisconsin, 1965, edited by R. H. Bing and R. J. Bean, Ann. of Math. Studies 60.

2. A. Flores, Uber die Existenz n-dimensionaler komplexe, die nicht in den $R_{2 n}$ topologisch einbettbar sind, Ergeb. math. Kolloq. 5 (1932/33), 17-24.

3. B.Grünbaum, Graphs and complexes, lecture notes, University of Washington, 1967.

4. — Imbedding of simplicial complexes, (to appear).

5. L. V. Keldysh, On embedding certain monotone images of $E^{n}$ in $E^{n}$ and $E^{n+1}$, Mat. 
Sb. 57 (1962), 95-104 (Russian).

6. C. Kuratowski, Sur le problème des courbes gauches en topologie, Fund. Math. 15 (1930), 271-283.

7. T. M. Price, A necessary condition that a cellular upper semicontinuous decomposition of $E^{n}$ yield $E^{n}$, Trans. Amer. Math. Soc. 122 (1966), 427-435.

8. D. E. Sanderson, Isotopy in 3-manifolds I, Isotopic deformation of 2-cells and 3cells, Proc. Amer. Math. Soc. 8 (1957), 912-922.

9. J. H. C. Whitehead, Simplicial spaces, nuclei, and m-groups, Proc. London Math. Soc. 45 (1939), 243-327.

10. J. Zaks, On a minimality property of complexes, Proc. Amer. Math. Soc. (to appear). 11. E. C. Zeeman, Seminar on combinatorial topology, Institut des hautes etudes scientifiques, 1963.

Received May 14, 1968. Research supported in part by the National Science Foundation, Grant GP-7536. The results which are presented in this paper are part of the author's dissertation, written at the University of Washington under the directions of Professor B. Grünbaum.

UNIVERSITY OF WASHINGTON

Seattle, Washington 



\section{PACIFIC JOURNAL OF MATHEMATICS}

\section{EDITORS}

\author{
H. ROYDEN \\ Stanford University \\ Stanford, California \\ R. R Phelps \\ University of Washington \\ Seattle, Washington 98105
}

\author{
J. Dugundui \\ Department of Mathematics \\ University of Southern California \\ Los Angeles, California 90007
}

RICHARD ARENS

University of California

Los Angeles, California 90024

\section{ASSOCIATE EDITORS}
E. F. BeCKENBACH
B. H. NeUmanN
F. WolF
K. YosidA

\section{SUPPORTING INSTITUTIONS}

\author{
UNIVERSITY OF BRITISH COLUMBIA \\ CALIFORNIA INSTITUTE OF TECHNOLOGY \\ UNIVERSITY OF CALIFORNIA \\ MONTANA STATE UNIVERSITY \\ UNIVERSITY OF NEVADA \\ NEW MEXICO STATE UNIVERSITY \\ OREGON STATE UNIVERSITY \\ UNIVERSITY OF OREGON \\ OSAKA UNIVERSITY \\ UNIVERSITY OF SOUTHERN CALIFORNIA
}

\author{
STANFORD UNIVERSITY \\ UNIVERSITY OF TOKYO \\ UNIVERSITY OF UTAH \\ WASHINGTON STATE UNIVERSITY \\ UNIVERSITY OF WASHINGTON

$* * * * *$
AMERICAN MATHEMATICAL SOCIETY
CHEVRON RESEARCH CORPORATION
TRW SYSTEMS
NAVAL WEAPONS CENTER

The Supporting Institutions listed above contribute to the cost of publication of this Journal, but they are not owners or publishers and have no responsibility for its content or policies.

Mathematical papers intended for publication in the Pacific Journal of Mathematics should be in typed form or offset-reproduced, double spaced with large margins. Underline Greek letters in red, German in green, and script in blue. The first paragraph or two must be capable of being used separately as a synopsis of the entire paper. It should not contain references to the bibliography. Manuscripts, in duplicate if possible, may be sent to any one of the four editors. Please classify according to the scheme of Math. Rev. 36, 1539-1546. All other communications to the editors should be addressed to the managing editor, Richard Arens, University of California, Los Angeles, California, 90024.

50 reprints are provided free for each article; additional copies may be obtained at cost in multiples of 50 .

The Pacific Journal of Mathematics is published monthly. Effective with Volume 16 the price per volume (3 numbers) is $\$ 8.00$; single issues, $\$ 3.00$. Special price for current issues to individual faculty members of supporting institutions and to individual members of the American Mathematical Society: $\$ 4.00$ per volume; single issues $\$ 1.50$. Back numbers are available.

Subscriptions, orders for back numbers, and changes of address should be sent to Pacific Journal of Mathematics, 103 Highland Boulevard, Berkeley, California, 94708.

PUBLISHED BY PACIFIC JOURNAL OF MATHEMATICS, A NON-PROFIT CORPORATION

Printed at Kokusai Bunken Insatsusha (International Academic Printing Co., Ltd.), 7-17. Fujimi 2-chome, Chiyoda-ku, Tokyo, Japan. 


\section{Pacific Journal of Mathematics}

Vol. 28, No. 3

May, 1969

Jon F. Carlson, Automorphisms of groups of similitudes over $F_{3} \ldots \ldots \ldots$

W. Wistar (William) Comfort, Neil Hindman and Stelios A. Negrepontis,

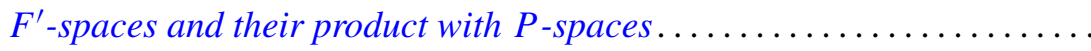

Archie Gail Gibson, Triples of operator-valued functions related to the unit

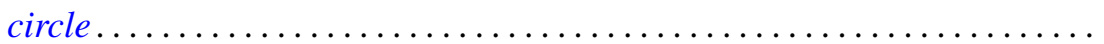

David Saul Gillman, Free curves in $E^{3}$

E. A. Heard and James Howard Wells, An interpolation problem for

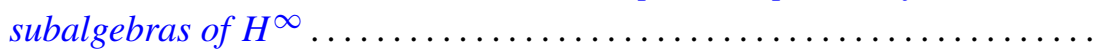

Albert Emerson Hurd, A uniqueness theorem for weak solutions of symmetric

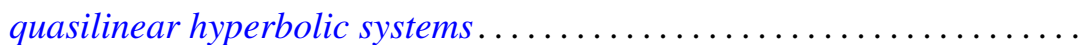

E. W. Johnson and J. P. Lediaev, Representable distributive Noether

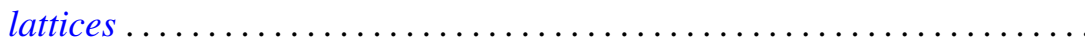

David G. Kendall, Incidence matrices, interval graphs and seriation in

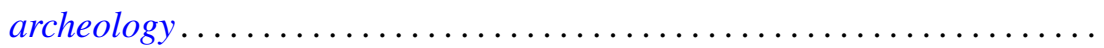
565

Robert Leroy Kruse, On the join of subnormal elements in a lattice ....... 571

D. B. Lahiri, Some restricted partition functions; Congruences modulo 3 .... 575

Norman D. Lane and Kamla Devi Singh, Strong cyclic, parabolic and conical differentiability........................................

William Franklin Lucas, Games with unique solutions that are

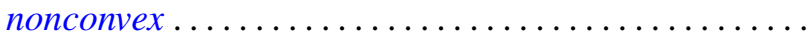

Eugene A. Maier, Representation of real numbers by generalized geometric series.

Daniel Paul Maki, A note on recursively defined orthogonal polynomials ...

Mark Mandelker, $F^{\prime}$-spaces and z-embedded subspaces ...

James R. McLaughlin and Justin Jesse Price, Comparison of Haar series with gaps with trigonometric series

Ernest A. Michael and A. H. Stone, Quotients of the space of irrationals ....

William H. Mills and Neal Zierler, On a conjecture of Golomb ...

J. N. Pandey, An extension of Haimo's form of Hankel convolutions ...

Terence John Reed, On the boundary correspondence of quasiconformal mappings of domains bounded by quasicircles...

Haskell Paul Rosenthal, A characterization of the linear sets satisfying Herz's criterion.

George Thomas Sallee, The maximal set of constant width in a lattice...

I. H. Sheth, On normaloid operators

James D. Stasheff, Torsion in BBSO ...

Billy Joe Thorne, A - P congruences on Baer semigroups.

Robert Breckenridge Warfield, Jr., Purity and algebraic compactness for

modules... 